\title{
The Predictive Value of Glomerular Filtration Rate-Based Scaling of Pediatric Clearance and Doses for Drugs Eliminated by Glomerular Filtration with Varying Protein-Binding Properties
}

\author{
Sinziana Cristea ${ }^{1}$ Elke H. J. Krekels ${ }^{1} \cdot$ Karel Allegaert ${ }^{2,3,4}$. Catherijne A. J. Knibbe ${ }^{1,5}$
}

Published online: 21 April 2020

(c) The Author(s) 2020

\begin{abstract}
Introduction For drugs eliminated by glomerular filtration (GF), clearance (CL) is determined by GF rate (GFR) and the unbound fraction of the drug. When predicting CL of GF-eliminated drugs in children, instead of physiologically based pharmacokinetic (PBPK) methods that consider changes in both GFR and protein binding, empiric bodyweight-based methods are often used. In this article, we explore the predictive value of scaling using a GFR function, and compare the results with linear and allometric scaling methods for drugs with different protein-binding properties.

Methods First, different GFR maturation functions were compared to identify the GFR function that would yield the most accurate GFR predictions across the pediatric age range compared with published pediatric inulin/mannitol CL values. Subsequently, the accuracy of pediatric CL scaling using this GFR maturation function was assessed and compared with PBPK CL predictions for hypothetical drugs binding, to varying extents, to serum albumin or $\alpha$-acid glycoprotein across the pediatric age range. Additionally, empiric bodyweight-based methods were assessed.

Results The published GFR maturation functions yielded comparable maturation profiles, with the function reported by Salem et al. leading to the most accurate predictions. On the basis of this function, GFR-based scaling yields reasonably accurate (percentage prediction error $\leq 50 \%$ ) pediatric CL values for all drugs, except for some drugs highly bound to AGP in neonates. Overall, this method was more accurate than linear or 0.75 allometric bodyweight-based scaling.

Conclusion When scaling CL and dose by GFR function, maturational changes in plasma protein concentrations impact GF minimally, making this method a superior alternative to empiric bodyweight-based scaling.
\end{abstract}

Electronic supplementary material The online version of this article (https://doi.org/10.1007/s40262-020-00890-2) contains supplementary material, which is available to authorized users.

Catherijne A. J. Knibbe

c.knibbe@antoniusziekenhuis.nl

1 Division of Systems Biomedicine and Pharmacology, Leiden Academic Center for Drug Research, Leiden University, Leiden, The Netherlands

2 Department of Development and Regeneration, KU Leuven, Leuven, Belgium

3 Department of Pharmaceutical and Pharmacological Sciences, KU Leuven, Leuven, Belgium

4 Clinical Pharmacy, Erasmus MC Rotterdam, Rotterdam, The Netherlands

5 Department of Clinical Pharmacy, St. Antonius Hospital, Nieuwegein, The Netherlands

\section{Key Points}

A maturation function for glomerular filtration is preferred for scaling clearance (CL) and doses from adults to the pediatric population over empiric bodyweightbased methods.

Maturation in the expression of drug binding plasma proteins and associated changes in unbound drug fractions has limited influence on pediatric CL and dose scaling, except for $\alpha$-acid glycoprotein-bound drugs in neonates.

Our findings are relevant for defining (first-in-child) doses in clinical studies, particularly for drugs for which differences in dose requirements between adults and children can be attributed entirely to differences in pharmacokinetics. 


\section{Introduction}

Clearance (CL) is the driving parameter for dosing as it determines steady-state and trough concentrations. For children, precise scaling of CL without bias across the pediatric age range is paramount to reach both an effective and safe (starting) dose. This is of relevance for defining (first-in-child) doses in clinical studies, particularly for drugs for which differences in dose requirements between adults and children can be attributed entirely to differences in pharmacokinetics (PK) and/or for which target concentrations in children are known [1].

CL of drugs eliminated through glomerular filtration (GF) is dependent on the GF rate (GFR) and plasma protein binding. GFR maturation across the pediatric population has been described by different functions based on data from CL of either endogenous (e.g. creatinine, cystatin C) or exogenous (e.g. inulin, iohexol, aminoglycosides) compounds, used as markers for GFR function [2-7]. With respect to plasma protein binding, changes in the unbound drug fraction $\left(f_{\mathrm{u}}\right)$ with age need to be taken into account when predicting pediatric CL via GF, as only the drug fraction that is not bound to plasma proteins can be eliminated through GF. The unbound fraction across age is dependent on the protein the drug binds to (i.e. human serum albumin or $\alpha$-acid glycoprotein [AGP]) and the changes in the concentrations of these proteins with age [8]. As physiologically-based PK (PBPK) models include drug properties (i.e. $f_{\mathrm{u}}$ ) and physiological differences between adults and children (i.e. maturation of plasma protein concentrations and GFR), these models are considered the 'gold standard' for pediatric CL predictions [9].

However, the application of PBPK approaches is constrained by the availability of drug-specific data, skilled personnel, and resources needed to access and use different modeling platforms. Therefore, empirical bodyweightbased scaling methods such as linear scaling or allometric scaling with a fixed exponent of 0.75 are still often used to derive pediatric CL from adult CL values. However, empirical scaling methods disregard information about maturation of both GFR and protein binding. Previous work has shown that these approaches are inaccurate for certain pediatric age groups for drugs cleared by GF [10, 11], suggesting that more mechanistic information may be needed for accurate scaling. For this, it has been proposed to adjust the allometric scaling with a maturation function for GFR, especially in the very young [12]. In this article, we assess the accuracy of scaling based on GFR maturation, without taking into account maturational changes in $f_{\mathrm{u}}$. We compare this approach with two relatively straightforward scaling methods based on bodyweight alone, since these methods are still often used and are perhaps even preferred because of their ease.

To this end, we first identified the GFR maturation function that yields the most accurate GFR predictions across the pediatric age range. Subsequently, we assessed the accuracy of pediatric CL and dose scaling obtained with the GFR maturation function compared with PBPK predictions for hypothetical drugs binding, to varying extents, to human serum albumin (HSA) or AGP across the pediatric age range. Additionally, the results are compared with those of the two empiric bodyweight-based methods, i.e. linear and allometric scaling with a fixed exponent of 0.75 .

\section{Methods}

\subsection{Establishing the Most Accurate Pediatric Glomerular Filtration Rate (GFR) Maturation Function}

Functions that quantify GFR maturation throughout the pediatric age range for children with normal renal functionality, and that only used demographic characteristics as input, were collected from the literature by searching the PUBMED database using the search term "glomerular filtration maturation children human", or from Simcyp v18 resources. Seven [7, 13-17] functions were identified, of which six [13-17] were developed based on exogenous markers for GFR (i.e. inulin, -Cr-EDTA, mannitol, iohexol) and one [7] was derived from $C L$ values of antibiotics that are predominantly eliminated through GF. To visually compare the different GFR maturation profiles, age-appropriate body surface area (BSA), height, and weight values were derived from the National Health and Nutrition Examination Survey (NHANES) database [18] and used for GFR predictions with each of the seven functions.

In this analysis, inulin and mannitol CL values were considered the 'gold standard' for GF function $[19,20]$, and hence were used to select the most accurate pediatric GFR maturation function. GFR predictions with each of the seven maturation functions were compared with the inulin [3-6] and mannitol [2] CL values published for children, for whom the necessary demographic characteristics were reported. Individual data were either digitized using WebPlotDigitizer (https://apps.automeris.io/wpd/) or extracted directly from the publications. When inulin and mannitol CL values were reported relative to the standard adult BSA (i.e. normalized by $1.73 \mathrm{~m}^{2}$ ), they were converted to absolute values. When gestational age was missing, a gestational period of 38 weeks was imputed. Missing BSA values were calculated based on age and bodyweight using the Haycock et al. [21] and Dubois et al. [22] formulas for children under and over $15 \mathrm{~kg}$, respectively. 
For the seven GFR maturation functions, the demographic characteristics corresponding to the individuals for whom inulin $[3,4,6]$ and mannitol [2] CL values were available were used as input, and the resulting predictions were compared with the reported measurements. For this, a percentage prediction error $\left(\% \mathrm{PE}_{\mathrm{GFR}}\right)$ between the predicted GFR with each function and the inulin $[3,4,6]$ and mannitol [2] CL values was calculated according to Eq. (1). In addition, the root mean square percentage error $\left(\% \mathrm{RMSPE}_{\mathrm{GFR}}\right)$ was calculated using Eq. (2), for the entire pediatric population as well as selected age groups, to show the stratified accuracy of the GFR functions for preterm neonates, term neonates on the first day, newborns aged between 1 day and 1 month, and children aged between 1 and 6 months, 6 months and 1 year, 1 and 5 years, and between 5 and 15 years. In Eqs. (1) and (2), the predicted GFR are values obtained with each of the published GFR maturation functions, and observed $\mathrm{CL}_{\text {inulin/mannitol }}$ are the published values for inulin or mannitol CL.

$\% \mathrm{PE}_{\mathrm{GFR}}=\frac{\text { predicted } \mathrm{GFR}-\text { observed } \mathrm{CL}_{\text {inulin/manitol }}}{\text { observed } \mathrm{CL}_{\text {inulin/manitol }}} \times 100$

\subsection{Evaluation of Pediatric Clearance (CL) Scaling}

To evaluate the accuracy of pediatric CL scaling using the selected GFR function or empiric functions, a 'true' CL value is needed as reference. As PBPK-based approaches are considered the 'gold standard' for pediatric CL predictions, the renal PBPK model in Eq. (3) was used to derive 'true' CL values. 'True' CL of hypothetical drugs was predicted for typical pediatric individuals at ages 1 day, 1, 3, 6 and 9 months, 2, 5, 10, and 15 years, and a 35-year-old typical adult.

'true' CL $=$ GFR $\times f_{\mathrm{u}}$

In Eq. (3), pediatric GFR values were obtained using the best maturation function selected above. Demographic values needed to predict pediatric GFR values with the best GFR maturation function were derived from the NHANES database [18] and the International Commission on Radiological Protection (ICRP) annals [24] for children and adults, respectively.

For $f_{u}$ in Eq. (3), a total of 20 hypothetical drugs were evaluated. For these drugs, $f_{\mathrm{u}}$ values in adults $\left(f_{\mathrm{u} \text {,adult }}\right)$ of $0.1,0.2,0.3$, $0.4,0.5,0.6,0.7,0.8,0.9$, or 1 were used and each drug was assumed to exclusively bind to either HSA or AGP. Pediatric $f_{\mathrm{u}}$ values $\left(f_{\mathrm{u} \text {,ped }}\right)$ at each pediatric age were obtained based on

$\% \mathrm{RMSPE}_{\mathrm{GFR}}=\sqrt{\frac{1}{n} \times \sum_{i=1}^{n}\left(\frac{\text { predicted } \mathrm{GFR}-\text { observed } \mathrm{CL}_{\text {inulin/manitol }}}{\text { observed } \mathrm{CL}_{\text {inulin/manitol }}}\right)^{2}}$

As the predictions do not include variability or uncertainty in any of the terms, only point estimates of $\% \mathrm{PE}_{\mathrm{GFR}}$ and $\% \mathrm{RMSPE}_{\mathrm{GFR}}$ are obtained. To compensate for this, rather than applying the twofold rule that is commonly used in assessing the accuracy of PBPK model prediction, we designated values within $\pm 30 \%$ to be 'accurate predictions', values outside the $\pm 50 \%$ interval to be 'inaccurate', and values in between to be 'reasonably accurate' for $\% \mathrm{PE}_{\mathrm{GFR}}$. For $\% \mathrm{RMSPE}_{\mathrm{GFR}}$, values within 0-30\% indicate 'accurate predictions', values $>50 \%$ indicate 'inaccurate predictions', and values within $30-50 \%$ are 'reasonably accurate'. The GFR maturation function that would lead to the narrowest range in $\% \mathrm{PE}_{\mathrm{GFR}}$ predictions and the smallest $\% \mathrm{RMSPE}_{\mathrm{GFR}}$ overall and per age group was selected and used in the PBPK-based approach, as well as in the evaluation of pediatric CL scaling.

The results here do not include findings for preterm neonates, as only four [7, 13, 15] of the seven GFR maturation functions were also developed for preterm neonates. Inulin and mannitol data collected from preterm neonates $[3,5,23]$ were analyzed separately, together with these four functions, and the results can be found in the electronic supplementary material (ESM). the ratios between relevant binding protein concentrations and the $f_{\text {u,adult }}$, according to Eq. (4) [8]:

$f_{\text {u,ped }}=\frac{1}{1+\frac{[P]_{\text {ped }} \times\left(1-f_{\text {uadult }}\right)}{[P]_{\text {adult }} \times f_{\text {uadult }}}}$

where $[P]$ represents the plasma concentration of the relevant binding protein (i.e. HSA or AGP).

Equations (5) and (6) [15] were used to calculate the plasma concentrations $([P])$ of HSA and AGP, respectively, for typical children of different ages, with age expressed in days. Visual representations of the maturation profiles of the plasma proteins, as well as of the resulting $f_{\text {u,ped }}$ values, are presented in electronic supplementary Fig. S1.

$[\operatorname{HSA}(g / L)]=1.1287 \times \ln ($ Age $)+33.746$

$[\operatorname{AGP}(g / L)]=\frac{0.887 \times \operatorname{Age}^{0.38}}{8.89^{0.38}+\operatorname{Age}^{0.38}}$

where $[\mathrm{HSA}(g / L)]$ and $[\operatorname{AGP}(g / L)]$ represent the plasma protein concentrations, and Age is the age of the typical child expressed in days [15]. 


\subsection{GFR-based Scaling of CL}

For GFR-based scaling of CL from adults to children of different ages Eq. (7) is used. Here 'true' adult CL values of the drug, i.e. GFR adult multiplied by $f_{\text {u, adult }}$ (for 20 hypothetical drugs, see Eq. 3), were scaled by the ratio between GFR $_{\text {ped }}$ and GFR adult $_{\text {, with }}$ GFR $_{\text {ped }}$ calculated according to the selected function (see results, Salem et al. [17], Eq. 12). Note that $f_{\mathrm{u} \text {, adult }}$ is included for obtaining the 'true' adult CL values; however, changes in $f_{\mathrm{u}}$ with age are not included when applying GFR-based scaling (Eq. 7).

GFR scaled $\mathrm{CL}_{\text {ped }}={ }^{\prime}$ true' $\mathrm{CL}_{\text {adult }} \times\left(\frac{\mathrm{GFR}_{\text {ped }}}{\mathrm{GFR}_{\text {adult }}}\right)$

\subsection{Empiric and Linear Body-Weight Based Scaling Methods}

For comparative purposes, the accuracy of GFR-based scaling was evaluated together with linear bodyweightbased scaling (Eq. 8) and bodyweight-based allometric scaling with a fixed exponent of 0.75 (Eq. 9), which are two commonly used empirical pediatric CL scaling methods.

Linear scaled $\mathrm{CL}_{\text {ped }}={ }^{\prime}$ true ${ }^{\prime} \mathrm{CL}_{\text {adult }} \times\left(\frac{\mathrm{WT}_{\text {ped }}}{\mathrm{WT}_{\text {adult }}}\right)$

Allometric scaled $\mathrm{CL}_{\text {ped }}={ }^{\prime}$ true ${ }^{\prime} C L_{\text {adult }} \times\left(\frac{\mathrm{WT}_{\text {ped }}}{\mathrm{WT}_{\text {adult }}}\right)^{0.75}$

\subsection{Comparison of Different Scaling Methods}

The accuracy of CL obtained with GFR-based, linear, and allometric scaling with a fixed exponent of 0.75 was assessed by calculating the $\% \mathrm{PE}_{\mathrm{CL}}$ as compared with the 'true' $\mathrm{Cl}_{\text {ped }}$, according to Eq. (10). Note that in 'true' $\mathrm{CL}_{\text {ped }}$ (Eq. 3 ) the changes in $f_{\mathrm{u}}$ with age are considered, according to Eqs. (4)-(6).

$\% \mathrm{PE}_{\mathrm{CL}}=\frac{\text { scaled } \mathrm{CL}_{\text {ped }}-{ }^{\prime} \text { true }^{\prime} \mathrm{CL}_{\text {ped }}}{\text { 'true' } \mathrm{CL}_{\text {ped }}} \times 100$

\subsection{Assessment of Pediatric Dose Scaling}

As CL scaling is commonly used as the basis for dose scaling, the implications of the different CL scaling methods on the accuracy of the dose adjustments derived from them were also assessed. For each of the 20 hypothetical drugs for which 'true' adult CL values (Eq. 3) were calculated, Eq. (11) was used to derive the pediatric dose.

dose $_{\text {ped }}=$ dose $_{\text {adult }} \times\left(\frac{\mathrm{CL}_{\text {ped }}}{\text { true' }^{\prime} \mathrm{CL}_{\text {adult }}}\right) \times 100$

where $\mathrm{CL}_{\text {ped }}$ refers to $\mathrm{CL}$ values obtained with either of the three simplified scaling methods (GFR-based scaling, linear scaling, or allometric scaling with a fixed exponent of 0.75 ) according to Eqs. (7), (8), and (9), respectively. This method assumes steady-state conditions (i.e. drug exposure is only dependent on dose and CL) and that the same drug target exposure (i.e. AUC) is applicable in children and adults. As relative dose adjustments were assessed, the adult dose was expressed as 1 .

The 'true' reference doses were obtained by replacing the $\mathrm{CL}_{\text {ped }}$ value in Eq. (11) with the 'true' $\mathrm{CL}_{\text {ped }}$ value (Eq. 3). The accuracy of the scaled doses was assessed by calculating the $\% \mathrm{PE}_{\text {dose }}$ according to Eq. (10).

\section{Results}

\subsection{Establishing the Most Accurate Pediatric GFR Maturation Function}

Figure 1 shows the seven published GFR maturation profiles [7, 13-17]. All profiles are comparable with the steepest maturation occurring in the first 2 years of life and plateau values being reached beyond the age of 15 years.

Figure 2 depicts the $\% \mathrm{PE}_{\mathrm{GFR}}$ between GFR predictions according to the seven different functions versus the inulin [3, 4, 6] or mannitol [2] CL measurements. In addition, Table 1 presents the $\% \mathrm{RMSPE}_{\mathrm{GFR}}$ and the range in $\% \mathrm{PE}_{\mathrm{GFR}}$ per age group, as well as for the entire pediatric age range. The results show that all functions tend towards overprediction of GFR in the very young. In newborns, interindividual variability is higher than in older children, which yields the largest spread in $\% \mathrm{PE}_{\mathrm{GFR}}$ for all GFR functions, with values ranging between -112 and 484\%. Furthermore, $\% \mathrm{RMSPE}_{\mathrm{GFR}}$ in newborns can reach values of $158 \%$ compared with values below $50 \%$ in older children. For all functions, the $\% \mathrm{PE}_{\mathrm{GFR}}$ range becomes narrower with increasing age, and, above 5 years, most functions lead to accurate predictions $\left(\% \mathrm{PE}_{\mathrm{GFR}}\right.$ within $\left.\pm 30 \%\right)$. The function reported by Salem et al. [17] had the best predictive performance per age group and across all pediatric ages. These GFR predictions were similar to those obtained using the function reported by Rhodin et al. [14], as indicated by the $\mathrm{RMSPE}_{\mathrm{GFR}} \%$ values and $\% \mathrm{PE}_{\mathrm{GFR}}$ ranges for the entire population, as well as for the different age groups. Results for preterm neonates are 

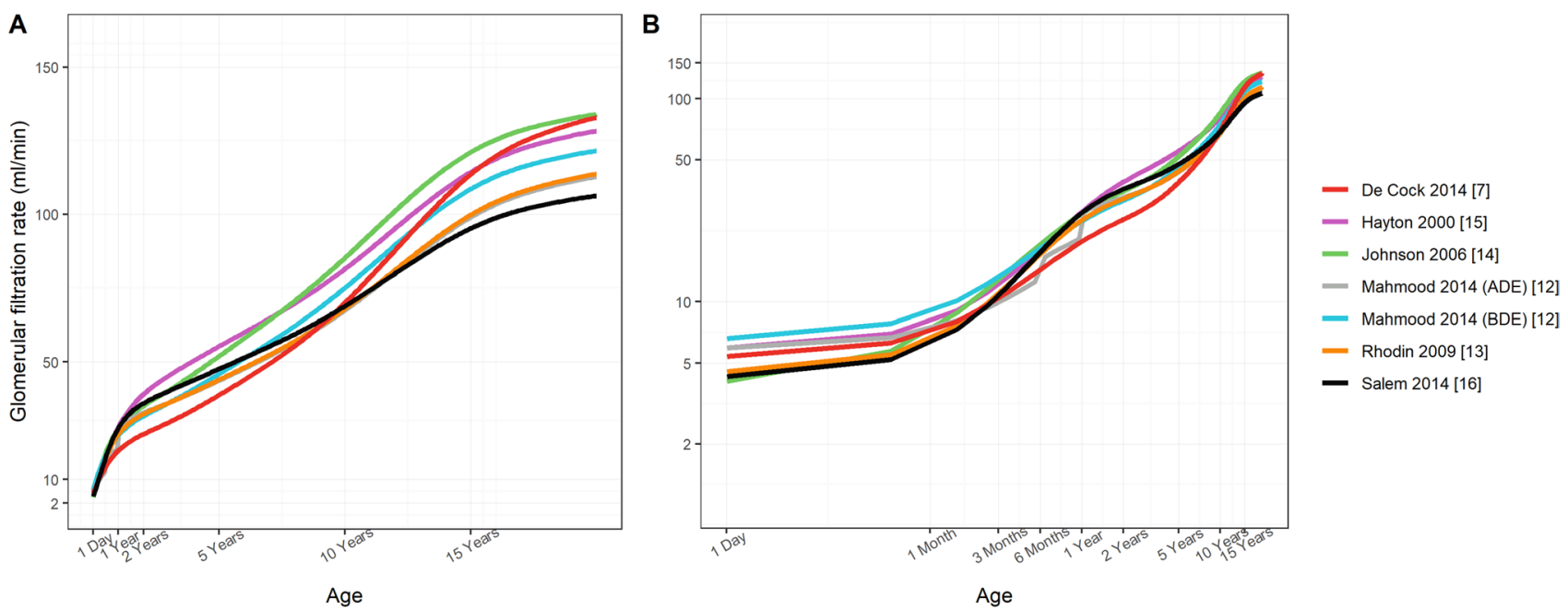

Fig. 1 Pediatric GFR according to published GFR maturation functions [7, 13-17] throughout the pediatric age range. a Semi-logarithmic scale; b double logarithmic scale. GFR glomerular filtration rate

presented in the ESM (electronic supplementary Fig. S2 and Table S1).

From these results, the GFR maturation function published by Salem et al. [17] (Eq. 12) was selected and used in the renal PBPK model (Eq. 3) to determine the 'true' renal $\mathrm{CL}$ of the 20 hypothetical drugs for the typical adult and the typical pediatric individuals. These GFR values are also used in Eq. (7) to calculate GFR-based scaled CL values across the pediatric range.

$\mathrm{GFR}_{\mathrm{ml} / \mathrm{min}}=112 \times\left(\frac{\text { Weight }(\mathrm{kg})}{70}\right)^{0.63} \times \frac{\mathrm{PMA}^{3.3}}{\mathrm{PMA}^{3.3}+\mathrm{TM}_{50}^{3.3}}$

where PMA is defined as postmenstrual age in weeks, and $\mathrm{TM}_{50}$ is defined as the PMA at which GFR reaches half the adult levels.

\subsection{Evaluation of Pediatric CL Scaling}

Figure 3 shows the $\% \mathrm{PE}_{\mathrm{CL}}$ for GFR-based scaling and for the two empirical bodyweight-based scaling methods, none of which take into account changes in plasma protein concentrations. The figure illustrates how scaling accuracy of CL with each of the three methods is impacted by $f_{\mathrm{u}}$ (color intensifies with increased $f_{\mathrm{u}}$ ) and plasma protein concentrations at every investigated age. Overall, GFR-based scaling is more accurate than the two empirical bodyweight-based methods, leading to $\% \mathrm{PE}_{\mathrm{CL}}$ values within $\pm 50 \%$ throughout the pediatric age range, except for children aged 1 day for drugs with high binding to AGP $\left(f_{\mathrm{u} \text { adult }}<0.3\right)$. Bodyweight-based allometric scaling with a fixed exponent of 0.75 is mostly inaccurate for individuals aged $<3$ months.
GFR-based scaling and linear scaling outperform allometric scaling for these subjects. For children between 6 months and 15 years of age, linear scaling is reasonably accurate, albeit with a trend in $\% \mathrm{PE}_{\mathrm{CL}}$ values, indicating systematic bias towards underprediction. In this age range, similar, yet less strong, trends are seen for allometric scaling with a fixed exponent of 0.75 , while GFR-based scaling is generally the most accurate of the three (Fig. 3).

\subsection{Assessment of Pediatric Dose Scaling}

Figure 4 and Table 2 show pediatric doses (expressed as a percentage of the adult dose) obtained using 'true' CL values versus those obtained using CL values upon scaling by the three simplified methods in typical patients for 20 hypothetical drugs differing in $f_{\mathrm{u}}$ in adults and binding to either HSA or AGP. Both the figure and table show that the 'true' doses predicted based on 'true' pediatric CL values are dependent on $f_{\mathrm{u}}$, whereas the scaled doses derived from CL values scaled using the three different simplified methods (i.e. GFR scaling, linear scaling, and allometric scaling) are not. Overall, the results show that doses obtained with GFR-based scaling are lower than the 'true' reference doses for drugs highly bound (i.e. $f_{\mathrm{u}}=0.1$ ) to HSA or AGP (up to $20-60 \%$, respectively). For drugs with low protein binding (i.e. $f_{\mathrm{u}}=0.9$ ), the differences between the 'true' reference dose and GFR-based scaled doses are small throughout the pediatric age range ( $<5 \%$ difference). Using linear bodyweightbased scaling, doses are also lower than the 'true' reference doses for children aged between 6 months and 10 years (up to $25.5-49 \%$ lower). For younger children, the difference between doses becomes smaller $(<30 \%$ difference). Doses obtained using bodyweight-based allometric scaling 

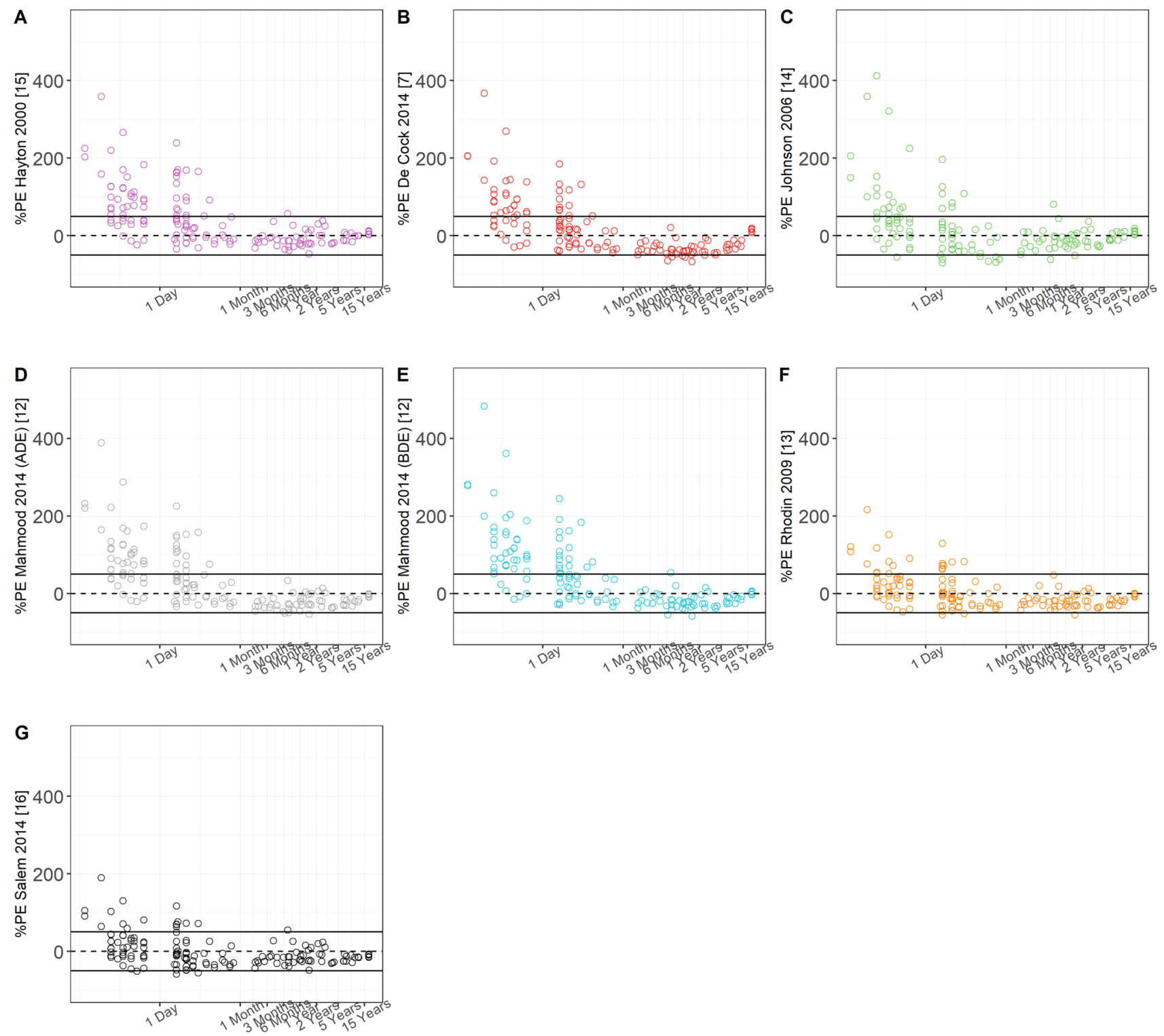

Fig. $2 \% \mathrm{PE}_{\mathrm{GFR}}$ between individual predictions, based on the seven published GFR maturation functions [7, 13-17] and individual literature data on inulin [3, 4, 6] and mannitol [2] clearance values versus age. The results for each published GFR maturation function are displayed in separate panels (a-g). The dashed line is the null-line, and

with a fixed exponent of 0.75 are generally higher than the 'true' reference doses for children younger than 6 months of age. For this method, the highest difference of $>150 \%$ was obtained for drugs with high fraction unbound in children younger than 1 month (Fig. 4, Table 2).

\section{Discussion}

This study aimed to identify the GFR maturation function that yields the most accurate GFR predictions across the entire pediatric age range, and to subsequently assess solid lines represent the $\% \mathrm{PE}_{\mathrm{GFR}}$ of $\pm 50 \%$ range that was considered to indicate reasonably accurate scaling. $\% P E_{G F R}$ percentage prediction error, GFR glomerular filtration rate, HSA human serum albumin, $A G P \alpha$-acid glycoprotein

what the accuracy of GFR-based scaling of CL and dose is compared with the "gold standard" (i.e. PBPK-based predictions) and with two commonly used empiric bodyweight-based scaling methods. By comparing scaled CL values with PBPK CL predictions, we studied the influence of the maturation of plasma protein concentrations on CL and dose scaling, and showed at what ages this maturation is of relevance for each scaling method. The assessed scaling methods are typically used to guide pediatric dosing when little or no information is available on a drug in this population. As such, this work identifies drug properties (i.e. $f_{\mathrm{u}}$ ) and patient characteristics (i.e. age) for 


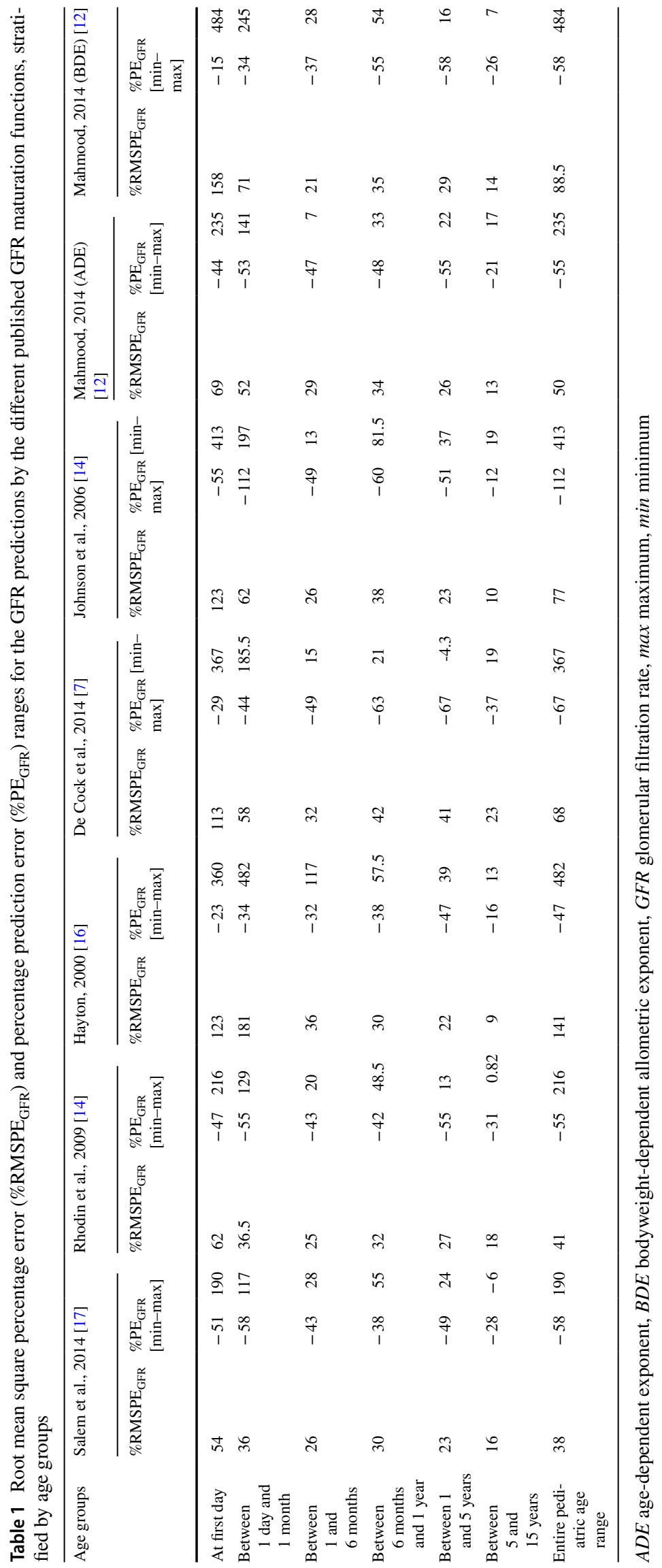




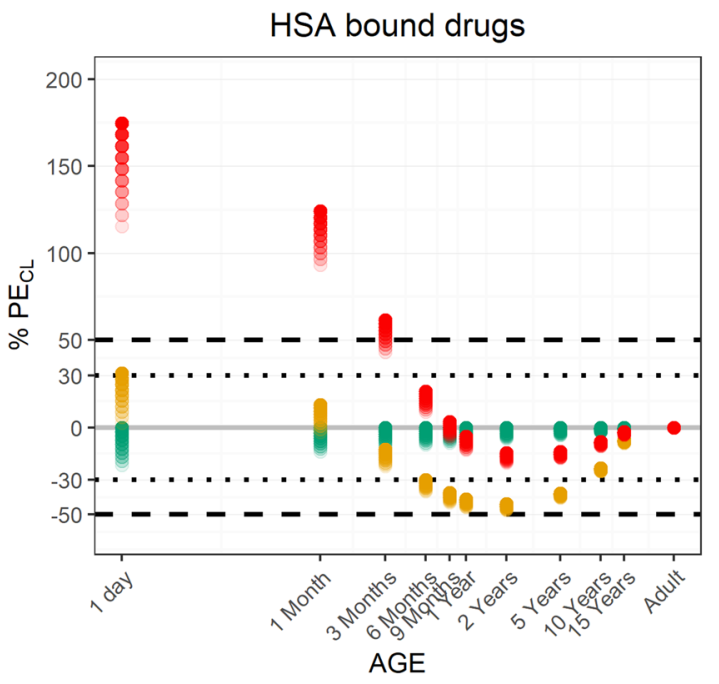

Fig. $3 \% \mathrm{PE}_{\mathrm{CL}}$ between 'true' $\mathrm{CL}$ values and $\mathrm{CL}$ values obtained using three different simplified scaling methods in typical pediatric patients for 20 hypothetical drugs differing in unbound drug fraction in adults and binding to either HSA (left panel) or AGP (right panel). Green dots indicate GFR-based scaling, orange dots indicate linear bodyweight-based scaling, and red dots indicate bodyweightbased scaling with a fixed allometric exponent of 0.75 . Colors

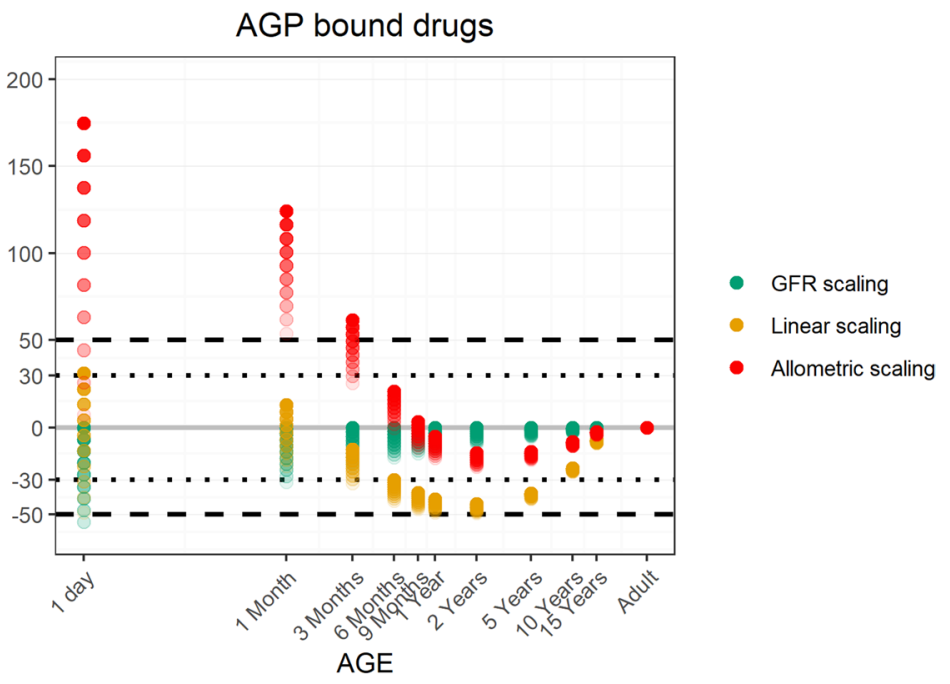

intensify with increasing $f_{\mathrm{u}}$. The grey solid line is the null-line, and black dashed lines and black dotted lines represent the $\% \mathrm{PE}_{\mathrm{CL}}$ range of $\pm 30 \%$ and $\pm 50 \%$, respectively, which indicate accurate and reasonably accurate scaling, respectively. $\% P E_{C L}$ percentage prediction error, $C L$ clearance, $G F R$ glomerular filtration rate, $f_{u}$ unbound drug fraction

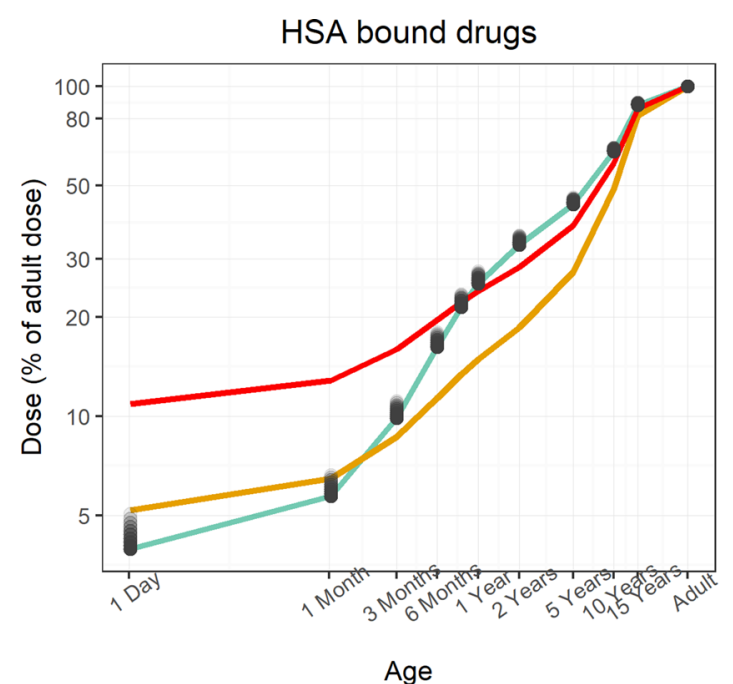

Fig. 4 Pediatric doses (a percentage of the adult dose) obtained with 'true' CL values (black dots) and CL values obtained with three different simplified scaling methods (lines) in typical pediatric patients for 20 hypothetical drugs differing in $f_{\mathrm{u}}$ in adults and binding to either HSA (left panel) or AGP (right panel). Green line indicates dose values obtained with GFR-based scaling, orange line indicates

which bodyweight-based scaling methods suffice and when more mechanistic information is necessary by means of either GFR-based scaling or PBPK for accurate CL and dose scaling. Our findings provide guidance for (first-inchild) clinical studies on what scaling method to use when

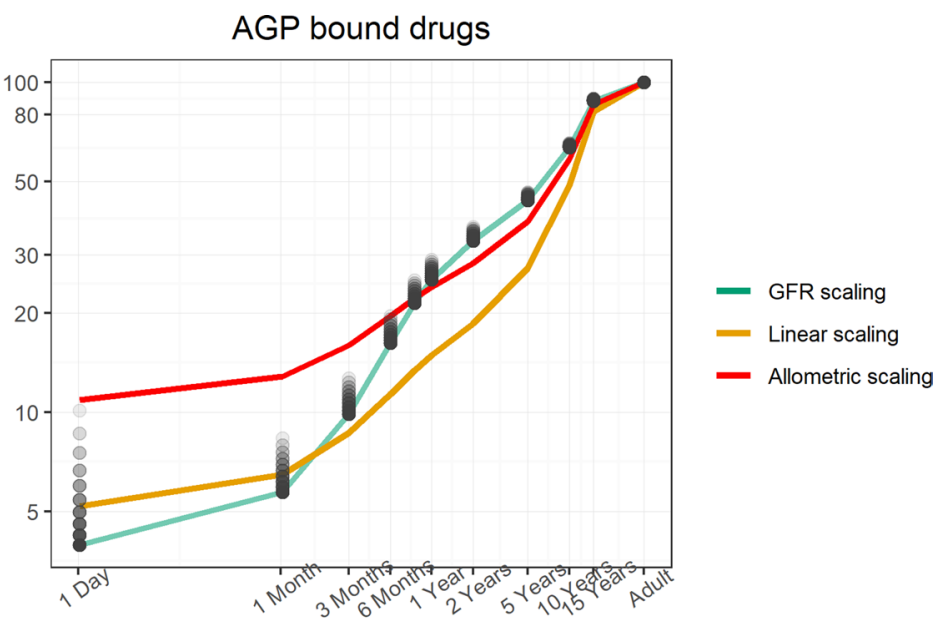

Age dose values obtained with linear bodyweight-based scaling, and red line indicates dose values obtained with bodyweight-based scaling with a fixed allometric exponent of 0.75 . The black dots indicate dose values obtained with 'true' CL. Color intensifies with increasing $f_{\mathrm{u}}$. $C L$ clearance, $f_{u}$ unbound drug fraction, $H S A$ human serum albumin, $A G P \alpha$-acid glycoprotein, GFR glomerular filtration rate

deriving pediatric doses from adult doses of small molecule drugs that are mainly eliminated by GF.

The published GFR maturation functions we evaluated were found to have comparable profiles, while the functions published by Salem et al. [17] and Rhodin et al. [14] had 
Table 2 Pediatric doses presented as $\%$ of adult dose for drugs eliminated through GFR with varying $f_{\mathrm{u}}$ values

\begin{tabular}{|c|c|c|c|c|c|c|c|c|c|}
\hline \multicolumn{3}{|c|}{$\begin{array}{l}\text { Demographic characteristics of typical } \\
\text { individuals }\end{array}$} & \multicolumn{4}{|c|}{$\begin{array}{l}\text { 'True' dose (\% of adult dose) obtained based on 'true' } \\
\text { CL }\end{array}$} & \multicolumn{3}{|c|}{$\begin{array}{l}\text { Scaled dose (\% of adult dose) obtained } \\
\text { using three CL scaling methods }\end{array}$} \\
\hline \multirow[t]{2}{*}{ Age } & \multirow[t]{2}{*}{ Weight $^{\mathrm{a}}(\mathrm{kg})$} & \multirow[t]{2}{*}{$\mathrm{GFR}^{\mathrm{b}}(\mathrm{mL} / \mathrm{min})$} & \multicolumn{2}{|c|}{ Drugs binding to HSA } & \multicolumn{2}{|c|}{ Drugs binding to AGP } & \multirow[t]{2}{*}{ GFR scaling (\%) } & \multirow{2}{*}{$\begin{array}{l}\text { Linear } \\
\text { scaling } \\
(\%)\end{array}$} & \multirow{2}{*}{$\begin{array}{l}\text { Allometric } \\
\text { scaling }(\%)\end{array}$} \\
\hline & & & $f_{\mathrm{u}}=0.1(\%)$ & $f_{\mathrm{u}}=0.9(\%)$ & $f_{\mathrm{u}}=0.1(\%)$ & $f_{\mathrm{u}}=0.9(\%)$ & & & \\
\hline 1 day & 3.4 & 4.3 & 5 & 4.1 & 10.1 & 4.2 & 4 & 5.2 & 11 \\
\hline 1 month & 4.3 & 6.2 & 6.6 & 5.8 & 8.3 & 5.9 & 5.7 & 6.5 & 13 \\
\hline 3 months & 5.8 & 10.7 & 11.1 & 10 & 12.7 & 10.1 & 9.9 & 8.6 & 16 \\
\hline 6 months & 7.5 & 17.6 & 17.9 & 16.4 & 19.6 & 16.5 & 16.2 & 11.4 & 20 \\
\hline 9 months & 8.9 & 23.2 & 23.5 & 21.6 & 25.1 & 21.8 & 21.4 & 13.4 & 22 \\
\hline 1 year & 9.9 & 27.4 & 27.5 & 25.5 & 29.1 & 25.6 & 25.3 & 14.9 & 24 \\
\hline 2 years & 12.3 & 35.9 & 35.4 & 33.3 & 36.5 & 33.4 & 33.1 & 18.6 & 28 \\
\hline 5 years & 18.2 & 47.7 & 46 & 44.2 & 46.6 & 44.3 & 44 & 27.4 & 38 \\
\hline 10 years & 32.5 & 68.9 & 65.4 & 63.8 & 65.6 & 63.8 & 63.6 & 48.9 & 58 \\
\hline 15 years & 54.2 & 95.3 & 89.7 & 88.1 & 89.7 & 88.1 & 87.9 & 81.6 & 86 \\
\hline Adult & 66.5 & 108.4 & 100 & 100 & 100 & 100 & 100 & 100 & 100 \\
\hline
\end{tabular}

The 'true' doses predicted based on 'true' pediatric CL values are dependent on $f_{\mathrm{u}}$ whereas the scaled doses derived from CL values scaled with the three different scaling methods (i.e. GFR scaling, linear scaling and allometric scaling) are not

$A G P \alpha$-acid glycoprotein, $C L$ clearance, $f_{u}$ unbound drug fraction, GFR glomerular filtration rate, HSA human serum albumin, ICRP International Commission on Radiological Protection, NHANES National Health and Nutritional Examination Survey

${ }^{a}$ Weights derived from (1) the NHANES database [18] for children, and (2) the ICRP annals [24] for adults

${ }^{\mathrm{b}}$ GFR values were obtained with Salem et al. [17]

similar accuracy in predicting inulin $[3,4,6]$ and mannitol [2] CL measures, with the function reported by Salem et al. [17] being slightly more accurate overall. This function (Eq. 12) was used in PBPK-based predictions of 'true' pediatric CL values (Eq. 3) and was directly used for simplified GFR-based scaling (Eq. 7).

Drug CL by GF depends on GFR and plasma protein binding, which are taken into account by PBPK modeling approaches. However, the extent of protein binding and the proteins the drugs bind to may not always be known, especially for the pediatric population. The simplified scaling functions, which include GFR-based scaling (Eq. 7), bodyweight-based linear scaling (Eq. 8), and bodyweight-based allometric scaling with a fixed exponent of 0.75 (Eq. 9), typically do not take into account changes in plasma protein binding with age. The difference between GFR-based scaled pediatric CL values and 'true' pediatric CL values reflects the impact of ignoring maturation in plasma protein concentrations on CL scaling. The current analysis showed that with GFR-based scaling, this impact can be disregarded throughout the entire pediatric age range, except in neonates for a few drugs highly bound to AGP (Fig. 3). Prediction errors in scaled CL values are largest in neonates, especially for drugs that bind to AGP, possibly due to the steep maturation of AGP plasma concentrations in early life (electronic supplementary Fig. S1). GFR-based scaling leads to underprediction of CL in neonates and in drug doses, compared with 'true' $C L$ and 'true' reference doses, which will result in not only a reduced risk of developing toxic effects but also an increased risk of treatment failure. Bodyweightbased allometric scaling with a fixed exponent of 0.75 tends to overpredict $C L$ in children younger than 6 months, even though for drugs with a low $f_{\mathrm{u}}$, maturational changes in the expression of drug binding plasma proteins can still partially correct this bias. Bodyweight-based linear scaling leads to reasonably accurate $\mathrm{CL}$ predictions in this young population. After the age of 6 months, the influence of plasma protein binding on CL scaling decreases, as shown by a smaller deviation of GFR-based scaled CL from PBPK-based CL predictions. In this age range, reasonably accurate CL predictions are obtained using bodyweight-based scaling, irrespective of whether the exponent is 1 (linear scaling), 0.75 (allometric scaling), or 0.62 (GFR function reported by Salem et al. [17]). As scaled CL values drive the scaled dose values, the same patterns are observed for this variable.

The CL predictions of selected drugs ( $>80 \%$ renal elimination) in neonates and children, using the GFR maturation function reported by Rhodin et al. [14], has recently been described [25]. Our results are in line with these published findings, with the added advantage that our analysis captures the entire hypothetical parameter space regarding the relevant drug-specific parameters (i.e. extent and type of plasma protein binding). As such, the presented analysis covers drugs that are currently in clinical use and other small molecule drugs that are still to be developed. Therefore, this framework can be used to make 
a priori assessments on the accuracy of the pediatric $\mathrm{CL}$ and dose-scaling methods for new drugs.

The current results are also in line with previous findings from our group comparing 'true' PBPK-based CL predictions with CL values scaled by both empirical methods; however, small differences in numerical results are present. These differences are caused by two different GFR maturation functions being used in the PBPK model for predictions of the 'true' CL values. For the current analysis, we used the function published by Salem et al. [17], which we found to be most accurate, whereas, in the previous analyses, the function by Johnson et al. [15] was used.

The conclusions from our analysis are based on typical individuals and do not take interindividual variability into account. For preterm and term neonates younger than 1 month, high variability in the inulin $[3,4,6]$ and mannitol [2] CL data is observed, which poses a challenge when scaling $C L$ and doses to this age range. This suggests that variables other than the demographics used in GFR maturation functions are predictive of GFR-based CL. For this special population, dosing recommendations that rely on empiric PK models of the same drug, even in slightly older children, or of a similar drug that is mainly eliminated through GF in the same population, may therefore offer a better alternative [26, 27].

We emphasize that all published GFR maturation functions included in our analysis describe GFR maturation in pediatric individuals with normal renal function. These functions should therefore not be used for CL or dose scaling for pediatric patients with renal deficiencies. To account for renal impairment, functions that require a biomarker for renal function (e.g. creatinine, cystatin $\mathrm{C}$, etc.) as input are more reliable and suitable to predict GFR. These functions can be implemented in the renal PBPK model in Eq. (3) and can also be used for GFR-based scaling. The impact of ignoring plasma protein binding in these scenarios may not be the same as observed in the current analysis, as plasma protein binding may also be altered in patients with renal deficiencies.

\section{Conclusion}

The maturation function reported by Salem et al. [17] (Eq. 12) describes GFR most accurately throughout the pediatric age range compared with data on inulin and mannitol CL. GFR-based CL and dose scaling for drugs eliminated through GFR yields reasonably accurate pediatric $C L$ and dose values, despite ignoring the influence of maturational changes in protein binding, except for drugs highly bound to AGP in neonates.
Acknowledgements The authors would like to thank Linda B.S. Aulin for performing the code review.

\section{Compliance with ethical standards}

Funding Catherijne A.J. Knibbe received support from the Innovational Research Incentives Scheme (Vidi grant, June 2013) of the Dutch Organization for Scientific Research (NWO) for the submitted work.

Conflict of interest S. Cristea, E.H.J. Krekels, K. Allegaert and Catherijne A.J. Knibbe declare they have no conflicts of interest.

Open Access This article is licensed under a Creative Commons Attribution-NonCommercial 4.0 International License, which permits any non-commercial use, sharing, adaptation, distribution and reproduction in any medium or format, as long as you give appropriate credit to the original author(s) and the source, provide a link to the Creative Commons licence, and indicate if changes were made. The images or other third party material in this article are included in the article's Creative Commons licence, unless indicated otherwise in a credit line to the material. If material is not included in the article's Creative Commons licence and your intended use is not permitted by statutory regulation or exceeds the permitted use, you will need to obtain permission directly from the copyright holder.To view a copy of this licence, visit http://creativecommons.org/licenses/by-nc/4.0/.

\section{References}

1. Dunne J, Rodriguez WJ, Murphy MD, Beasley BN, Burckart GJ, Filie JD, et al. Extrapolation of adult data and other data in pediatric drug-development programs. Pediatrics. 2011;128(5):e1242e12491249. https://doi.org/10.1542/peds.2010-3487.

2. Rubin MI, Bruck E, Rapoport M, Snively M, McKay H, Baumler A. Maturation of renal function in childhood: clearance studies. J Clin Invest. 1949;28:1144-62. https://doi.org/10.1172/JCI102149.

3. Coulthard MG. Maturation of glomerular filtration in preterm and mature babies. Early Hum Dev. 1985;11:281-92. https://doi. org/10.1016/0378-3782(85)90082-9.

4. Dean RFA, McCance RA. Inulin, diodone, creatinine and urea clearances in newborn infants. J Physiol. 1947;106(4):431-9.

5. Leake RD, Trygstad CW, Oh W. Inulin clearance in the newborn infant: Relationship to gestational and postnatal age. Pediatr Res. 1976;10:759-62. https://doi.org/10.1203/00006450-19760800000013.

6. Oh W, Oh MA, Lind J. Renal function and blood volume in newborn infant related to placental transfusion. Acta Paediatr. 1966;55:197-21010. https://doi.org/10.1111/j.1651-2227.1966. tb15226.x.

7. De Cock RFW, Allegaert K, Brussee JM, Sherwin CMT, Mulla H, De Hoog M, et al. Simultaneous pharmacokinetic modeling of gentamicin, tobramycin and vancomycin clearance from neonates to adults: towards a semi-physiological function for maturation in glomerular filtration. Pharm Res. 2014;31:2643-54. https://doi. org/10.1007/s11095-014-1361-z.

8. McNamara PJ, Alcorn J. Protein binding predictions in infants. AAPS PharmSci. 2002;4:E4. https://doi.org/10.1208/ps040104.

9. Edginton AN, Schmitt W, Willmann S. Development and evaluation of a generic physiologically based pharmacokinetic model for children. Clin Pharmacokinet. 2006;45(10):1013-34. https:// doi.org/10.2165/00003088-200645100-00005.

10. Krekels EHJ, Calvier EAM, van der Graaf PH, Knibbe CAJ. Children are not small adults, but can we treat them as such? CPT 
Pharmacometrics Syst Pharmacol. 2019;8(1):34-8. https://doi. org/10.1002/psp4.12366.

11. Calvier E, Krekels E, Valitalo P, Rostami-Hodjegan A, Tibboel D, Danhof M, et al. Allometric scaling of clearance in paediatrics: when does the magic of 0.75 fade? Clin Pharmacokinet. 2017;56:273-85. https://doi.org/10.1007/s40262-016-0436-x.

12. Anderson BJ, Holford NHG. Mechanistic basis of using body size and maturation to predict clearance in humans. Drug Metab Pharmacokinet. 2009;24:25-36. https://doi.org/10.2133/dmpk.24.25.

13. Mahmood I. Dosing in children: a critical review of the pharmacokinetic allometric scaling and modelling approaches in paediatric drug development and clinical settings. Clin Pharmacokinet. 2014;53:327-46. https://doi.org/10.1007/s40262-014-0134-5.

14. Rhodin MM, Anderson BJ, Peters AM, Coulthard MG, Wilkins B, Cole M, et al. Human renal function maturation: a quantitative description using weight and postmenstrual age. Pediatr Nephrol. 2009;24:67-766. https://doi.org/10.1007/s00467-008-0997-5.

15. Johnson TN, Rostami-Hodjegan A, Tucker GT. Prediction of the clearance of eleven drugs and associated variability in neonates, infants and children. Clin Pharmacokinet. 2006;45:931-56. https ://doi.org/10.2165/00003088-200645090-00005.

16. Hayton WL. Maturation and growth of renal function: dosing renally cleared drugs in children. AAPS PharmSci. 2000;2:E3. https://doi.org/10.1208/ps020103.

17. Salem F, Johnson TN, Abduljalil K, Tucker GT, Rostami-Hodjegan A. A re-evaluation and validation of ontogeny functions for cytochrome P450 1A2 and 3A4 based on in vivo data. Clin Pharmacokinet. 2014;53(7):625-36. https://doi.org/10.1007/s4026 2-014-0140-7.

18. National Health and Nutrition Examination Survey. Available at: www.cdc.gov/growthcharts/index.htm

19. Schwartz GJ, Furth SL. Glomerular filtration rate measurement and estimation in chronic kidney disease. Pediatr Nephrol. 2007;22(11):1839-48. https://doi.org/10.1007/s0046 7-006-0358-1.
20. Kiss K, Molnár M, Söndergaard S, Molnár G, Ricksten SE. Mannitol clearance for the determination of glomerular filtration rate-a validation against clearance of 51Cr-EDTA. Clin Physiol Funct Imaging. 2018;38(1):10-6. https://doi.org/10.1111/ cpf.12374.

21. Haycock GB, Schwartz GJ, Wisotsky DH. Geometric method for measuring body surface area: a height-weight formula validated in infants, children, and adults. J Pediatr. 1978;93(1):62-6. https ://doi.org/10.1016/S0022-3476(78)80601-5.

22. Du Bois D, Du Bois EF. Clinical calorimetry: tenth paper a formula to estimate the approximate surface area if height and weight be known. Arch Intern Med. 1916;2(6):863-71. https:// doi.org/10.1001/archinte.1916.00080130010002.

23. Barnett BHL, Hare K, Mcnamara H, Hare R. Measurement of glomerular filtration rate in premature infants. J Clini Investig. 1948;27(6):691-9.

24. Valentin J. Basic anatomical and physiological data for use in radiological protection: reference values. ICRP Publication 89: Approved by the Commission in September 2001. Ann ICRP. 2002;32:1-277. https://doi.org/10.1016/S0146-6453(03)00002-2.

25. Wang J, Kumar SS, Sherwin CM, Ward R, Baer G, Burckart GJ, et al. Renal clearance in newborns and infants: predictive performance of population-based modeling for drug development. Clin Pharmacol Ther. 2019;105(6):1462-70. https://doi.org/10.1002/ cpt.1332.

26. Krekels EHJ, Neely M, Panoilia E, Tibboel D, Capparelli E, Danhof M, et al. From pediatric covariate model to semiphysiological function for maturation: Part I-extrapolation of a covariate model from morphine to zidovudine. CPT Pharmacometrics Syst Pharmacol. 2012;1(10):e9. https://doi.org/10.1038/psp.2012.11.

27. Calvier EAM, Krekels EHJ, Yu H, Välitalo PAJ, Johnson TN, Rostami-Hodjegan A, et al. Drugs being eliminated via the same pathway will not always require similar pediatric dose adjustments. CPT Pharmacometrics Syst Pharmacol. 2018;7(3):175-85. https://doi.org/10.1002/psp4.12273. 